\title{
Interference Cancellation Method Based on LMS Filter for UFMC System
}

\author{
Yu Xiang, Wang Shan and Yang Lu Duan Sirui \\ Chongqing University of Posts and Telecommunications, 400065 \\ 657908130@qq.com
}

\begin{abstract}
As a $5 G$ candidate waveform, Universal Filtered Multi-Carrier(UFMC) technology supports asynchronous transmission., the UFMC systems is sensitive to the multipath fading channel as OFDM system. Since the multipath fading channel has inter-carrier interference (ICI) and inter-symbol interference (ISI), the performance of the system is decreased dramatically. This paper presents a method to eliminate these interferences in the UFMC system, based on the Least Mean Square algorithm(LMS) processing and input by $2 N$ point $F F T$ frequency domain signal. The signal error is close to zero in the receiver with the help of the iterative operation and filter processing, which can eliminate the interference caused by the multipath fading in the receiver. The simulation results show that the UFMC system has better bit error rate performance by means of the interference cancellation method.
\end{abstract}

Keywords: UFMC; LMS; multipath fading channel; inter-carrier interference; intersymbol interference

\section{Introduction}

For 2020 and the future, Machine Type Communications(MTC) and Internet of things(IoT) are considered as the main driving force of the development of mobile communications, providing a broad application prospects for the fifth generation of mobile communication $(5 \mathrm{G})$. Compared with the $4 \mathrm{G}$ technology, the $5 \mathrm{G}$ technology is able to integrate a variety of wireless access and make full use of the low and high frequency spectrum resources, greatly enhancing spectrum efficiency and achieving sustainable development of the mobile communication network[1]. The most important multi-carrier modulation technique is orthogonal frequency division multiplexing (OFDM) in the 4G technology, which are widely used in the LTE and MIMO. However OFDM system is vulnerable to the impact of ICI and ISI. In other words, while orthogonality is not fully guaranteed, the signal will lead to distortion, reducing the performance of the system[2].

The novel waveform will be applied to next generation wireless communication systems(5G), such as FBMC, UFMC, F-OFDM and FB-OFDM[3].All these new technologies are based on the filtering methods, improving signal performance and spectral efficiency compared with OFDM. Filter-bank based Multi-carrier relies on dividing the spectrum into multiple orthogonal sub-bands which applies a filtering functionality on a per sub-carrier basis. Thus the FBMC side-lodes become weaker than OFDM and inter-carrier interference(ICI) issue will be reduced. But the frequency response of the filter requires very long filter lengths. UFMC, which is known as UFOFDM, can be parameterized to OFDM[4]. It is known that FBMC performs a per subcarrier filtering and OFDM applies a filtering operation for the entire frequency band[5]. However, UFMC collecting the advantages FBMC is promising while avoiding its drawbacks, which applies a filtering functionality per sub-band instead of per sub-carrier or entire band[6]. This makes it advantageous for communication in short uplink bursts 
and asynchronous transmission, as required in potential application scenarios of $5 \mathrm{G}$ systems[7].Lack of cyclic prefix $(\mathrm{CP})$ in the UFMC systems, the synchronization and channel estimation become challenging tasks. The delay speed effect of multipath fading channel causes inter-carrier interference and inter-symbol interference[8].Due to the multipath fading channel and Doppler effect, the performance of UFMC system will be reduced dramatically. Thus it is essential to effectively reduce the interference and improve the transmission reliability which has become the focus in this field.

Up to now, the literature has predominantly addressed OFDM and OFMD/OQAM interference reduction. But no much more documents are about the adaptive interference suppression algorithm in UFMC system. A method based on adaptive filtering is proposed to eliminate the interference caused by carrier frequency offset in OFDM system[9]. Adaptive filter automatically adjusts the filter coefficient until the error is minimum, and finally the output signal is obtained at the receiver. [10] designed an least-meansquare(LMS) algorithm that each OFDM symbol estimates the complex coefficients by utilizing pilot signals and the received signal is added to each of the complex coefficient. After a number of overlapping operations, inter-carrier interference(ICI) and common phase error (CPE) is close to zero. Thus the proposed method can reduce the ICI. Because of no need for training sequences and symbols to be transmitted, there is no loss of bandwidth efficiency. Active Interference Cancellation(AIC) is widely used in the OFDM or OFDMA system.[11] discussed to reduce inter-sub-band interference by incorporating Active Interference Cancellation(AIC) into the UFMC system. But the method adopts the matrix and is more complex.

In this paper, an adaptive algorithm based on least mean square error (LMS) is proposed, which is essentially a statistical gradient algorithm for minimizing the mean square value of the received signal and the transmitted signal. The methods to eliminate the interference is utilizing LMS algorithm and adaptive filter. Through several iterations to update the filter coefficients and according to the value of the FFT output of the receiver, the error in the receiver is close to zero and finally to obtain the desired signal. The complexity of the algorithm is not high because of no matrix operations. Motivated by the proposed LMS, in this work, the step size is the maximum eigenvalue of the frequency domain signal at the receiver and varies according to the input frequency domain signal. Thus these interference can be eliminated caused by the multipath fading channel in the received signal, improving the bit error ratio(BER) performance of the UFMC system.

\section{UFMC System}

The structure of UFMC system is 


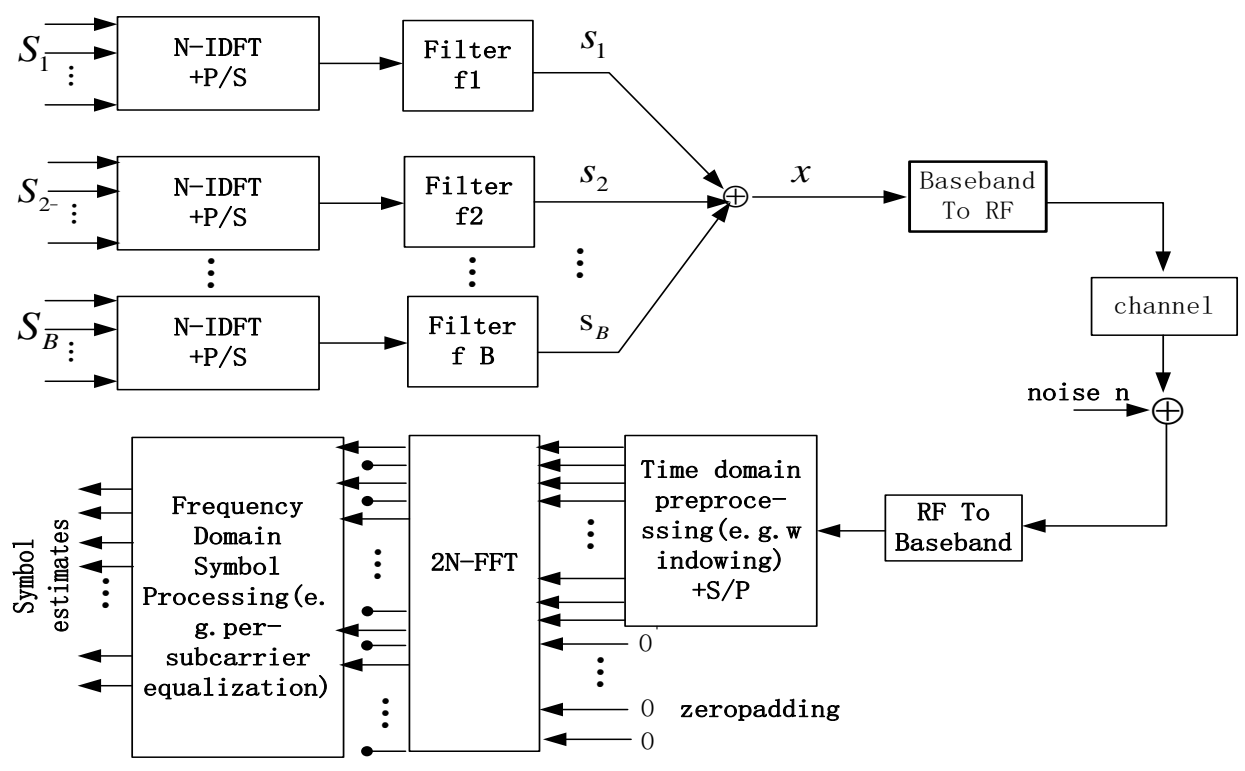

Figure 1. UFM//C Transceiver Architecture

In the UFMC system, the overall $K$ sub-carrier are divided into $\boldsymbol{B}_{\text {sub-bands }}$ and groups of sub-carrier are filtered. The number of sub-carriers on the sub-band $i$ is $K_{i}, K_{i} B=K$. Every sub-band is FFT point $N$.Thus the signals of the sub-band $i$ in frequency domain is converted into time domain transmit $s_{i}(n)$ by IDFT and can be expressed as

$$
\begin{aligned}
s_{i}(n) & =\frac{1}{N} \sum_{k=1}^{K_{i}} S_{i}(k) e^{j 2 \pi k n} / N \\
n & =0, \ldots, N-1
\end{aligned}
$$

Where $i$ is the sub-band index.

UFMC filters bundles of sub-carriers and each sub-band is filtered by the DolphChebyshev filter with the length of $L$. The filter can be same or different. Assuming that the filter of each sub-band is the same and with the length of $L$.Then the output signal $x_{i}$ of the sub-band $i$ can be written as

$$
\begin{aligned}
x_{i}(n) & =s_{i}(n) * f_{i}(n)=\sum_{l=0}^{L-1} f_{i} x_{i}(n-l) \\
n & =0, \ldots, N+L-1
\end{aligned}
$$

In the ides case, the discrete-time transmit signal of UFMC can be formulated as

$$
\begin{aligned}
x(n) & =\sum_{i=1}^{B} s_{i}(n) * f_{i}(n) \\
& =\sum_{i=1}^{B} \sum_{l=-\infty}^{\infty} s_{i} f_{i}(n-l) \\
& =\sum_{i=1}^{B} \sum_{l=-\infty}^{\infty}\left(\frac{1}{N} \sum_{k=1}^{K_{i}} S_{i}(k) e^{j 2 \pi k n / N}\right) f_{i}(n-l) \\
& =\sum_{i=1}^{B} \sum_{k=1}^{K_{i}} \sum_{l=-\infty}^{\infty} \frac{1}{N} \sum_{k=1}^{K_{i}} S_{i}(k) e^{j 2 \pi k n / N} f(n-l) \\
n & =0, \ldots, N+L-1
\end{aligned}
$$


Where $n$ and $l$ are time symbol index respectively. $S_{i}(k)$ is the $k_{\text {-th sub-carrier of }}$

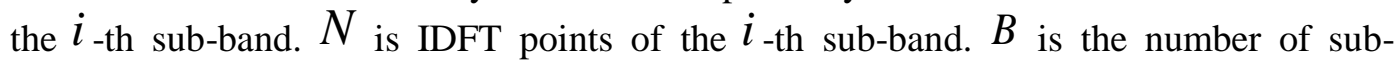
bands.

The signal transmits in the frequency selective fading channels. Thus discrete-time channel pules respond can be written as

$$
h=\left[h_{0}, h_{1 \ldots}, h_{V-1}\right]^{T}
$$

In the UFMC system, it is assumed that the signal is transmitted over a multipath fading channel. After $B$ sub-bands are filtered, the receive signal can be expressed as

$$
\begin{aligned}
y(n) & =\sum_{v=0}^{V-1} h(v) * x(n-v-\tau)+z(n) \\
& =\sum_{v=0}^{V-1} h(v) *\left(\sum_{i=1}^{B}\left(s_{i}(n-v-\tau) * f_{i}(n)\right)\right)+z(n)
\end{aligned}
$$

Where $z(n)$ is complex Gaussian noise with zero mean and variance $\sigma^{2}$.

At the receiver, zeros are padded in order to perform the 2N-point FFT. Thus the received time domain signal is converted into frequency by $2 \mathrm{~N}$-point FFT and given as

$$
Y(k)=\sum_{q=0}^{N+L-2} r(q) e^{-j 2 \pi k q} / 2 N, \quad k=0,1, \cdots, 2 N-1
$$

According to the characteristics of Fourier transform, (6) become

$$
\begin{aligned}
Y(k) & =\sum_{u=1}^{2 N-1} H(u) S_{i}(u) F(u) \\
& +\sum_{m=1, m \neq j}^{B} \sum_{u=0}^{2 N-1} H(u) S_{m}(u) F(u)+Z(k) \\
& =H(k) S_{i}(k) F(k)+I_{I C I}(k)+I_{I S I}(k)+Z(k)
\end{aligned}
$$

Because of multipath fading channel, inter-symbol interference(ISI) and inter-carrier interference(ICI) cannot be mitigated in the UFMC system .Form the above formula (7), it can be seen that signal is distorted in the UFMC system and its performance is down[14].Therefore, it is essential to eliminate the interference caused by the multipath fading channel and the Doppler effect in the UFMC system.

\section{Adaptive Interference Suppression Algorithm}

In order to eliminate the interference of UFMC system, it is needed to select the frequency domain signal and the reference signal. The structure of the proposed scheme is illustrated in Figure 2. 


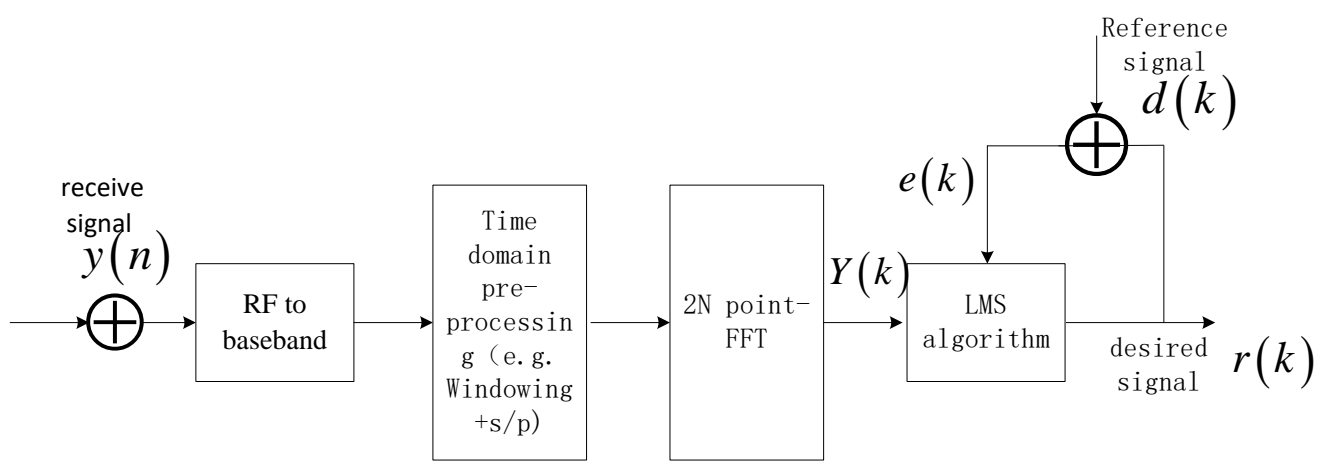

Figure 2. Receive Structure

As can be seen from the figure, the receiver of the UFMC system is added an adaptive filter based on LMS. The feature of LMS algorithm has been widely used in OFDM system, and the improved algorithm is applied to the UFMC system in this paper.

The LMS algorithm is a linear adaptive filtering algorithm, it can automatically adjust the filter coefficients according to the change of the input signal. Adaptive filters are widely used in system identification, interference cancellation and prediction. In this paper, the LMS adaptive filter is adopted to eliminate the interference caused by the multiple path channel in the UFMC system. Least mean square (LMS) and recursive least squares (RLS) are mostly used to update the filter coefficients adaptively. The adaptive filter adjusts its coefficients until it obtains the same desired signal as the reference signal, i.e. the signal has no error. Therefore, the adaptive filter can reduce the ICI and ISI caused by the channel by optimizing the filter coefficients.

The process of the interference cancellation of UFMC system using adaptive algorithm is as follows:

A. Firstly, the frequency domain signal $Y(k)$, the filter coefficient vector estimation value $^{w(k)}$ and the expected signal $d(k)$ are applied. Because of $r(k)$ and $d(k)$ are a stochastic process, the error signal $e(k)$ is also a stochastic process. We can write the error signal in

$$
\begin{aligned}
e(k) & =d(k)-r(k) \\
& =d(k)-W^{T}(k) Y(k)
\end{aligned}
$$

Where $Y(k)$ is the output of filter。 $d(k)$ is the transmitting of transmitter. $d(k)$ can be found by

$$
d(k)=\sum_{i=1}^{B} S_{i}(k)
$$

The output of the filter $r(k)$ is represented by the frequency domain $Y(k)$ signal and inner product of weight vector. So the $r(k)$ can be expressed as

$$
r(k)=W^{T}(k) Y(k)=Y^{T}(k) W(k)
$$

Where $T$ is the vector transpose.

According to the LMS algorithm, the minimized cost function is denote as 


$$
\zeta(k)=E\left(e_{k}(p) e_{k}^{*}(p)\right), p=1,2, \cdots
$$

Where $E_{\text {is expectation. The superscript }}{ }^{*}$ is conjugate.

In order to determine the adaptive filter coefficients of the $k$-th sub-carrier.It is required multiple iterations until the error signal is small enough.

B. The step size ${ }^{\mu}$ is used to estimate the weight of the filter weight by the LMS algorithm, initializing $W(k)=0$ at the first time. It is an important parameter, and the expression of the coefficients of the filter updated by the LMS algorithm is denote as

$$
W(k+1)=W(k)+2 \mu e(k) Y(k)
$$

The range of $\mu_{\text {value is defined as }}$

$$
0<\mu<1 / \lambda_{\max }
$$

Where $\lambda_{\max }$ is the largest eigenvalue of the $Y(k)$ auto correlation. From the definition of convergence, the size of the step size should be as small as possible, so that it converges to an optimum value. In the conventional LMS algorithm, the step size is fixed, while the step size in this paper is changed according to the frequency domain signal.

C. The output signal $y(k)$ is obtained after filtering. In order to obtain the desired output, the filter constantly adjusts the filter coefficient so that the mean square error reaches a minimum of 0 .

\section{Simulation Analysis}

After the theoretical analysis of the process of the adaptive algorithm, the simulations are performed to demonstrate the efficacy of the proposed scheme in this section. This section will adopt the MATLAB simulation software to analyze the performance of the interference which is caused by the multipath fading channel and LMS adaptive interference suppression algorithm. In the simulation, we compared UFMC with LMS algorithm and its performance. In order to better illustrate the usefulness of the proposed algorithm, the BER performance of UFMC and the proposed UFMC-LMS are compared under different channel conditions.

In the UFMC system simulation, the related parameters are as follow: FFT size of $N=1024$,filter length $L=20$ and the number of sub-bands $B=10$.The UFMC data symbols are modulated by QPSK technique. The number of sub-carriers in every subband is 12 . we assume that no guard sub-carriers between two consecutive sub-bands and all sub-bands are filtered by the same filters. The filter is Dolph-Chebyshev filter with $30 \mathrm{~dB}$ side lobe attenuation. The multipath fading and AWGN channel are considered. In the simulation process, the LMS equalization method is used to carry out the frequency domain equalization, which does not include the channel coding. Due to the influence of the multipath fading channel and the Doppler effect, the transmission signal will be distorted. Because of the adoption of the proposed LMS algorithm, interference can be eliminated which is caused by the channel in the UFMC system. As a result, the output of the system is substantially constant with the BER that is not subject to multipath fading channels. When the signal is transmitted in the 2-path fading channel and the input signal to noise ratio is $20 \mathrm{~dB}$, the constellation diagram of the UFMC system is 


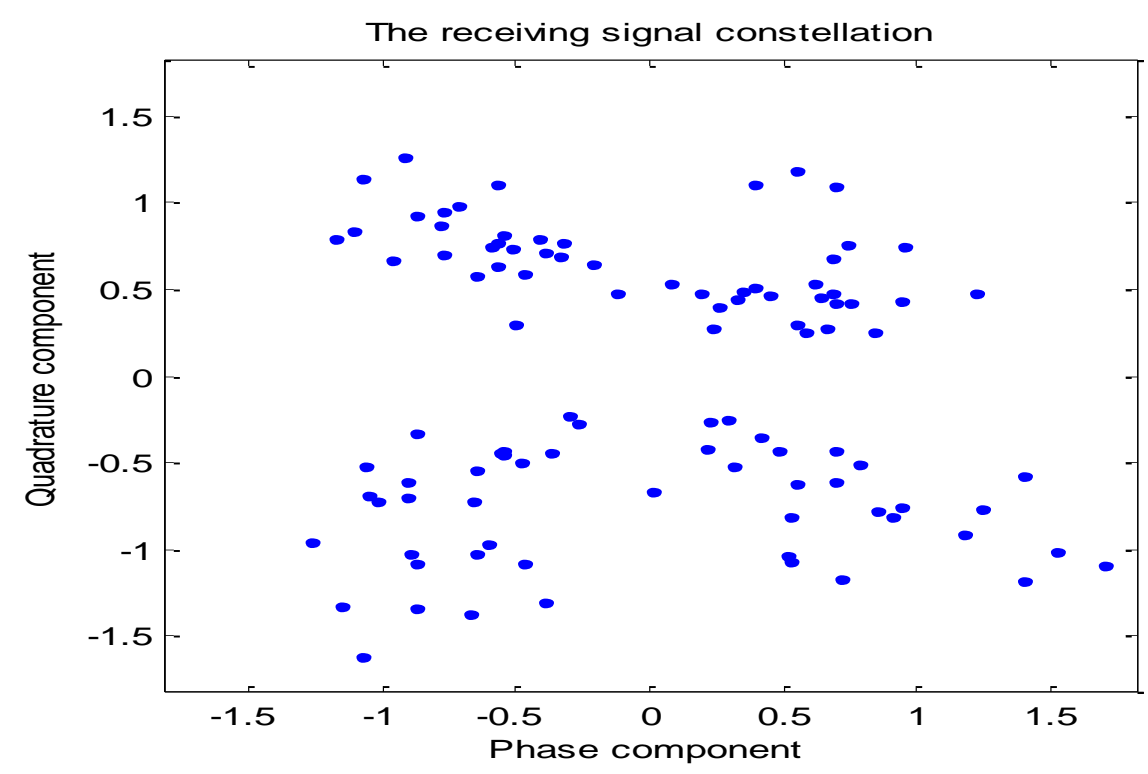

Figure 3. The Receiving Signal Constellation

As can be seen from Figure 3, the transmission signal is affected by the multipath fading channel and the constellation points have been dispersed. In the case of lower SNR, there will be a false decision. Therefore, in order to ensure the effective transmission of the signal and the interference cancellation of the signal at the receiver is worth studying. Due to the adoption of the proposed LMS algorithm, the interference can be eliminated caused by the channel in the UFMC system. Figure 4 shows the performance of UFMC system in different channel.

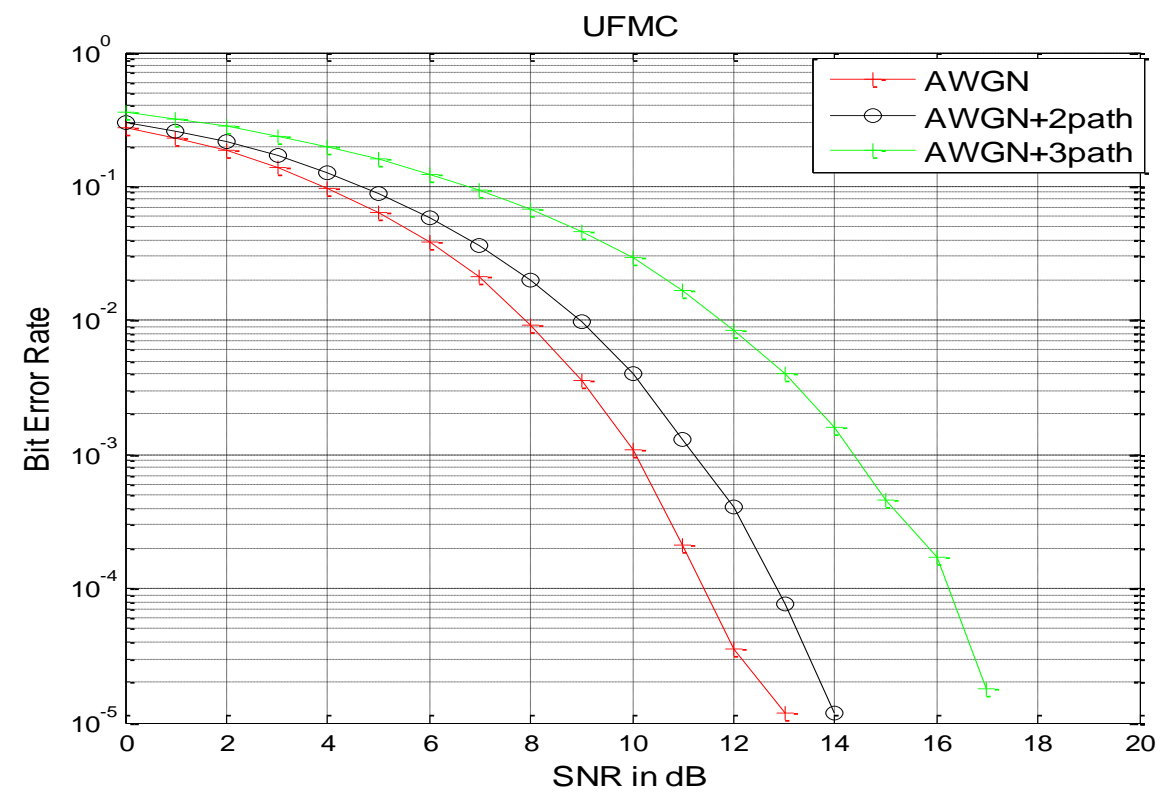

Figure 4. Performance of BER of UFMC System in Different Channel

It can be seen that the bit error rate increases with the increase of channel attenuation, the signal bit error rate increases. Thus it is necessary to reduce the interference of UFMC 
system. The simulation result of the proposed algorithm in different SNRs is illustrated in Figure 5.

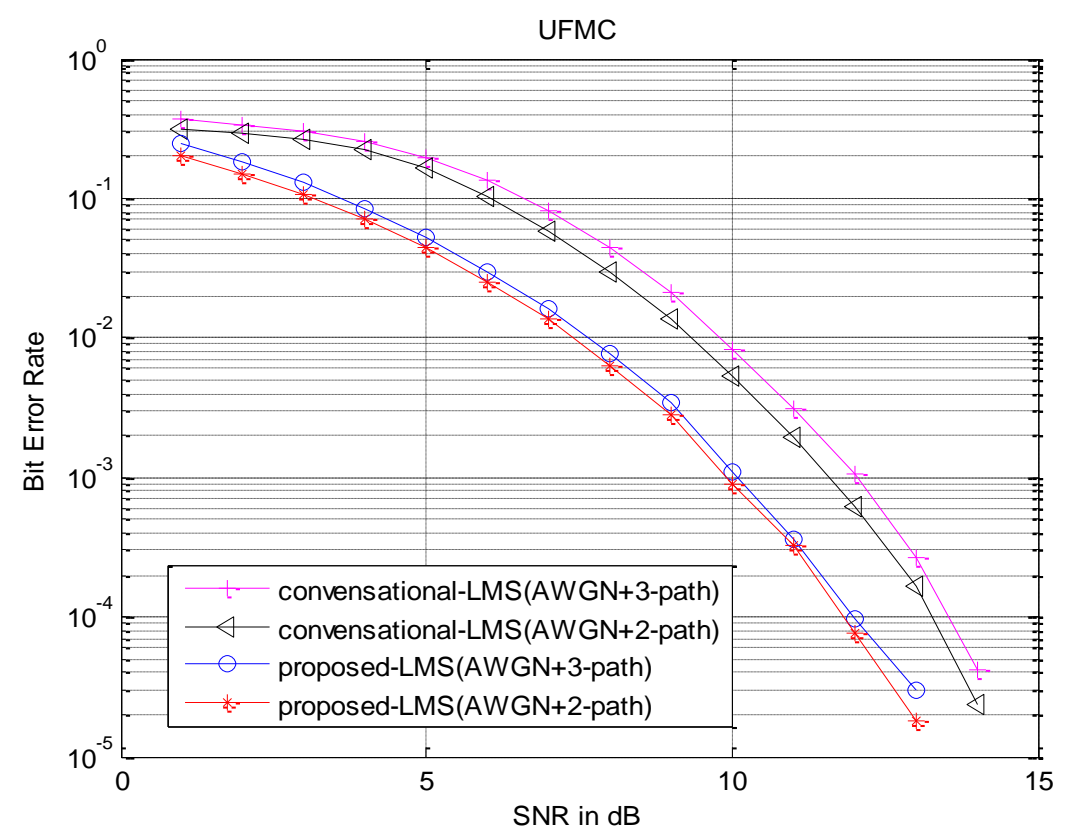

\section{Figure 5. Performance of Proposed LMS Algorithm in Different Channels}

Based on the Figure 5, it could be find that the proposed LMS algorithm is more better than the conventional LMS algorithm to reduce the ICI and ISI of UFMC system in the multiple fading channel. Thus the step size is the maximum eigenvalue of the frequency domain signal at the receiver, and the performance of the system will be better when the minimum value is taken.

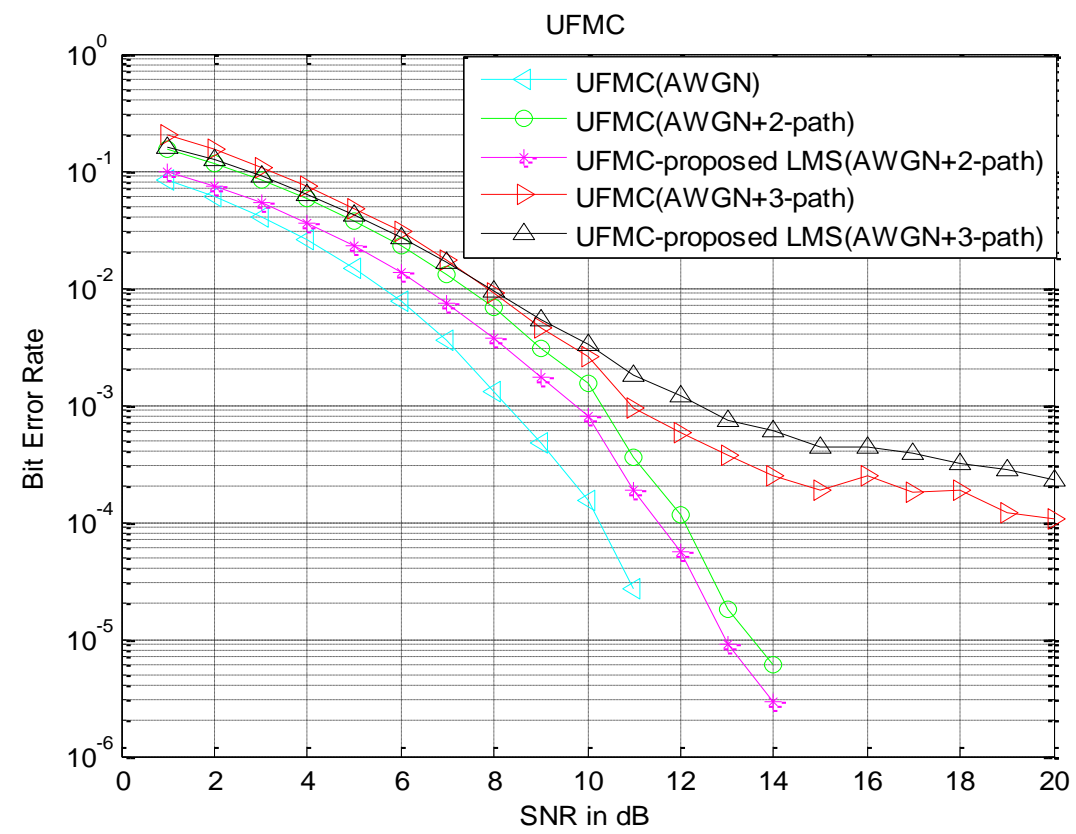

Figure 6. Performance of Interference Suppression of UFMC System in Different Channels 
With the increase of SNR, the proportion of ICI and IBI to the performance of system is increasing, but new method to improve the performance of the system is more and more obvious. From the above analysis, we can see that the proposed LMS algorithm in this paper can effectively restrain the interference caused by multiple fading channel under given simulation conditions.

\section{Conclusion}

In this paper, an adaptive interference suppression algorithm is discussed for a novel 5G candidate waveform technology of UFMC modulated by QPSK, and the performance of the system is analyzed in the multiple fading channel. It can be found in the simulation that the performance of UFMC system is improved in multipath channel. Detection and removal of interference in channel are important points to be solved in UFMC system. This adaptive filtering technique based on the LMS algorithm turn to eliminate the interference caused by the multipath channel. The simulation results show that the proposed frequency domain interference suppression method can avoid the interference energy leakage, effectively reduce the impact of interference, and the bit error rate of system has a significant improvement.

\section{Acknowledgements}

Fund Project: TD-LTE private network broadband multimedia cluster system equipment development and scale networking application verification (Major national science and technology projects 2015ZX03004004.

\section{References}

[1] G. Wunder, P. Jung, M. Kasparick, T. Wild, F. Schaich, Y. Chen, S.T. Brink, I. Gaspar, N. Michailow, A. Festag, L. Mendes, N. Cassiau, D. Ktenas, M. Dryjanski, S. Pietrzyk, B. Eged, P. Vago, F. Wiedmann, "5GNOW: non-orthogonal, asynchronous waveforms for future mobile applications", Communications Magazine, IEEE, vol.52,no.2, (2014), pp.97,105.

[2] G. Wunder, P. Jung, M. Kasparick, T. Wild, "5GNOW: non-orthogonal, asynchronous waveforms for future mobile applications", Communications Magazine, (2014), pp. 97-105.

[3] IMT-2020 ( 5G ) PG-White Paper On 5G Concept[OL]. URL: [2015-02-13]. http://www.imt2020.cn/zh/documents/listByQuery?currentPage $=1 \&$ content $=$.

[4] F.Schaich, T.Wild, Waveform contenders for 5G-OFDM vs. FBMC vs. UFMC[J], Proceedings of 6th International Symposium on Communications, Control, and Signal Processing(ISCCSP 2014), Athens, Greece,May 2014,in press, 2014:457-460.

[5] F. Schaich and T. Wild, "Relaxed Synchronization Support of Universal Filtered Multi-Carrier including Autonomous Timing Advance," ISWCS Workshop on Advanced Multi-Carrier Techniques for Next Generation Commercial and Professional Mobile Systems,Aug. 26-29, 2014.

[6] Wild, T, Shaich, F, Yejian Chen. 5G air interface design based on Universal Filtered (UF-)OFDM[C], Proceedings of the 19th International Conference on Digital Signal Processing, Aug.2014:699-704.

[7] J.Andrews, S. Buzzi, W. Choi, S. Hanly, A. Lozano, A. Soong, and J. Zhang. What will 5G be?[J]IEEE J. Select. Areas Commun,vol. 32, no. 6, pp. 1065-1082, June 2014:1065-1082.

[8] Wang X, Wild T, Schaich F, Pilot-Aided Channel Estimation for Universal Filtered MultiCarrier[C]//IEEE Vehicular Technology Conference (VTC Fall). 2015 IEEE 82nd. Boston, USA: IEEE Press, 2015: 1-5.

[9] A. R. Khedkar, pt. of Electron. \& Telecommun., Trained adaptive filter based approach to mitigate ICI in OFDM system[C], Pervasive Computing (ICPC), 2015: 1-4.

[10] K. Matsumoto, Y. Chang, Frequency Domain Phase Noise Compensation Emloying Adaptive Algorithms for Millimeter-Wave OFDM Systems[C], Microwave Conference (APMC), 2014: 12621264.

[11] H. Wang, Z. Zhang, "Universal filtered multi-carrier transmission with active interference cancellation", IEEE Wireless Communications \& Signal Processing(WCSP), (2015), pp. 1-6.

[12] M. Ghanbarisabagh , M. Y. Alias , H. A. Abdul-Rashid, Performance analysis of Least Mean Square Time-Domain Equalizer in long-haul direct-detection optical OFDM transmission[C]. Photonics (ICP), (2010), pp. 1-4.

[13] Sh.Tang, Ke.Gong, J.Song, Intercarrier interference cancellation with frequency diversity for OFDM systems[C]. IEEE Trans. Broadcast, (2007), 53: 132-137. 
International Journal of Future Generation Communication and Networking Vol.10, No.5 (2017)

[14] Wang, Xiaojie, Wild, Thorsten, Universal Filtered Multi-Carrier with Leakage-Based Filter Optimization[J], European Wireless, (2014), pp. 1-5. 